\title{
On-line species-specific isotopic analysis of sulfur by hyphenation of capillary electrophoresis with $\mathrm{MC}$ - ICP-MS
}

\author{
S. FASSBENDER ${ }^{1 *}$, K. RODIOUCHKINA ${ }^{2}$, F. VANHAECKE ${ }^{2}$ \\ AND B. MEERMANN ${ }^{1}$ \\ ${ }^{1}$ Federal Institute for Materials Research and Testing, \\ Inorganic Trace Analysis Div.,12489, Berlin, Germany \\ (*correspondence: sebastian.fassbender@bam.de) \\ ${ }^{2}$ Ghent University, A\&MS, 9000, Ghent, Belgium
}

In many scientific fields, isotopic analysis can offer valuable information. Up to date, typically bulk analysis is aimed at measuring the isotopic composition of the entire elemental content of the sample. However, the analyte element is usually present under the form of different species [1]. Thus, separating species of interest from one another and from matrix components prior to isotope ratio measurements can provide species-specific isotopic information [2, 3], which could be used for tracing the origin of environmental pollutants and elucidation of (environmental) speciation. Using on-line hyphenations of separation techniques with multicollector-ICP-MS (MC-ICP-MS) can save time and effort and enables the analysis of different species during a single measurement. Whereas some works hyphenating GC and IC with MC-ICP-MS have already been reported, LC and $\mathrm{CE}$ hyphenations are still inadequately represented based on the capabilities of these separation techniques.

In this work, we developed an on-line hyphenation of $\mathrm{CE}$ with multicollector-ICP-MS (CE/MC-ICP-MS) for isotopic analysis of sulfur species using a multiple-injection approach for instrumental mass bias correction by standard-sample bracketing. With this method, the isotopic composition of sulfur in sulfate originating from river water could be analyzed without sample preparation. The results were compared to data from off-line analysis of the same samples to ensure accuracy. The precision of the results of the on-line measurements was promising regarding the differentiation of the river systems by the isotopic signature of river water sulfate. The great potential of this method is based on the versatility of the applied separation technique, not only in the environmental field but also for, e.g., biomolecules, as sulfur is the only covalently bound constituent of proteins that can be analyzed by MC-ICP-MS.

[1] Rodriguez-Gonzalez et al. (2012) Mass Spectrom. Rev. 31, 504-521. [2] Moldovan et al. (2004) J. Anal. At. Spectrom. 19, 815-822. [3] Epov et al. (2010) Anal. Chem. 82, 5652-5662. 